\title{
Auer rod-like inclusions in a monoclonal B lymphocytosis
}

\author{
Lymphocytose B monoclonale \\ avec des inclusions de type corps d'Auer
}

\author{
Astrid Engelman ${ }^{1}$ \\ Delphine Manzoni ${ }^{1}$ \\ Jean-Claude Bordet ${ }^{1}$ \\ Liliana Vila ${ }^{1,2}$ \\ ${ }^{1}$ Laboratoire d'hématologie, \\ $\mathrm{CHU}$ de Lyon, France \\ ${ }^{2}$ Centre de lutte contre le cancer, \\ Centre Léon Bérard, Lyon, France
}

Article received December 27, 2018,

accepted May 15, 2019

A 91-year-old woman with no history of hematology disease was admitted to the emergency room for acute hip pain without obvious triggering factor. This patient was treated by a direct oral anticoagulant for atrial fibrillation.

CT scan revealed no abnormalities. The diagnosis of sciatica was retained. Her biology report was almost normal except for a lymphocytosis at $5.2410^{9} / \mathrm{L}$.

May-Grünwald-Giemsa-stained peripheral blood smear analysis showed monomorphic small lymphocytes with clumped chromatin, scant cytoplasm and some smudge cells typical of chronic lymphocytic leukemia (CLL) cells. Surprisingly, numerous CLL cells contained cytoplasmic Auer rod-like inclusions (figure $1 A, C$ ). These ones were also seen in smudge CLL cells (figure 1C). These atypicals lymphocytes represented $10 \%$ of total lymphocytes count. The cytoplasmic needle-shaped inclusions (figure 1D). were negative for myeloperoxidase by immunohistochemistry (myeloperoxydase kit RAL diagnostic ref.361610).

The immunological profile was in favor of CLL cells with Matutes score 5/5, since they expressed CD5/CD23/CD43/CD200 and showed weak expression of CD20/CD22/CD79b and surface immunoglobulin (figure $1 G$ ). FMC7 was negative. The monoclonal population representing $3.710^{9} / \mathrm{L}$, the diagnosis of high-count monoclonal B lymphocytosis was proposed. Fluorescence in situ hybridization revealed $13 q 14.3$ deletion without alterations of 11q, 12 or $17 \mathrm{p}$ chromosomes.

In electron transmission microscopy, the lymphocyte inclusions appeared as crystalline structures of 0.4 to 0.6 micrometers characteristic of immunoglobulin clusters, with in longitudinal sections a periodic density of $5 \mathrm{~nm}$ (figure 1E,F). The presence of Auer rod-like inclusions in lymphoid cell have already been described in rare case of chronic lymphoproliferative disorders $[1,2]$.

The diagnosis approach was similar in the two other publications we found [1, 2], underlying that even if we know that Auer rod-shaped inclusions aren't pathognomonic of myeloid malignancy, it can be beneficial to complement the cytological analysis with other test suchs as immunophénotyping, cytochemistry and even electron transmission microscopy to assess this very unusual phenomenon

In this case the careful examination of the cells during a night shift lead to the right diagnosis, as the cell's chromatin was clumped, mature and fortunately unmistakably lymphocyte-like. . . However the presence of Auer rod-like inclusions encouraged us to perform additional studies, and the use of electron microscopy lead to identifying the crystalline inclusions as aggregated immunoglobulin. 


\section{Biological pictures}

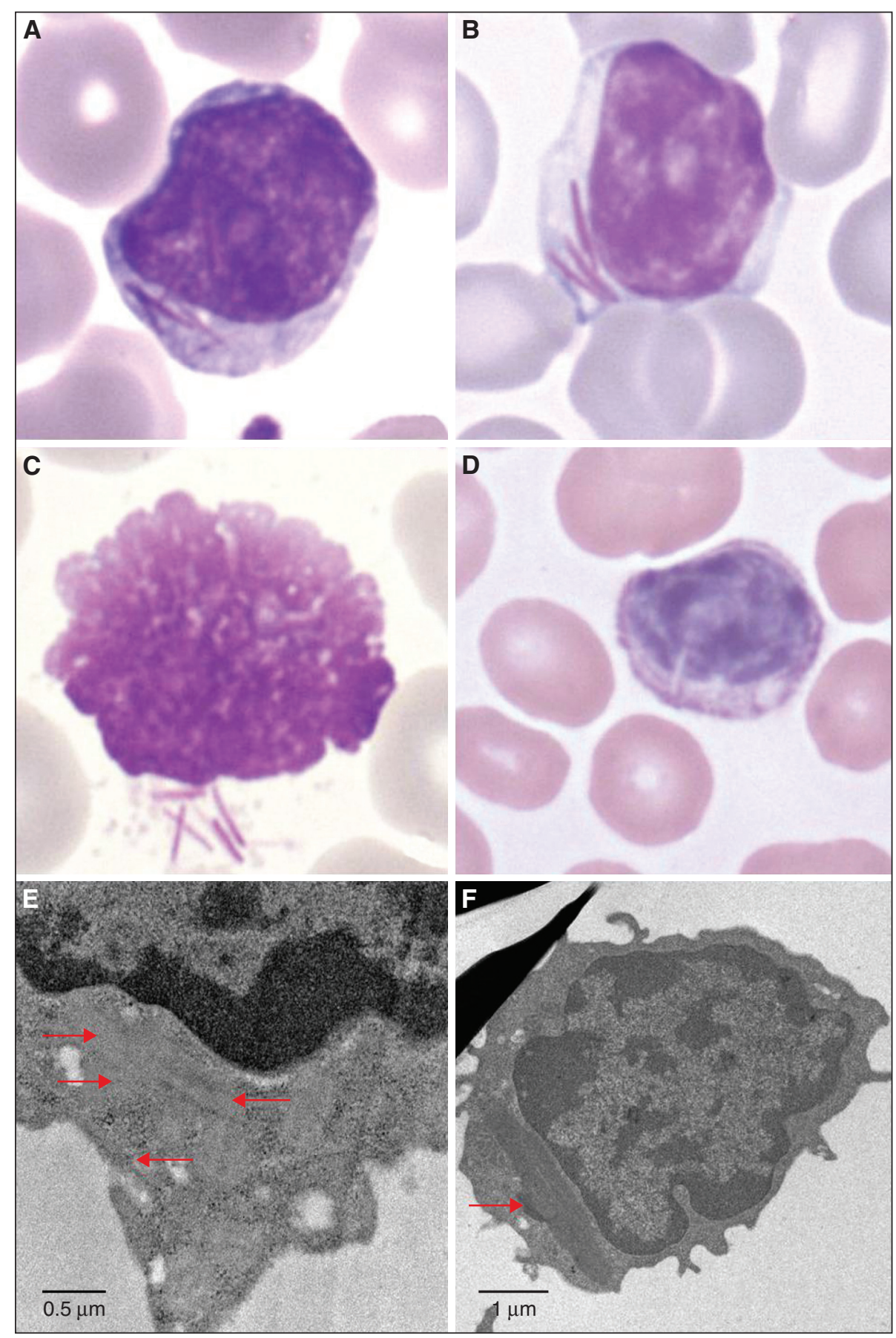




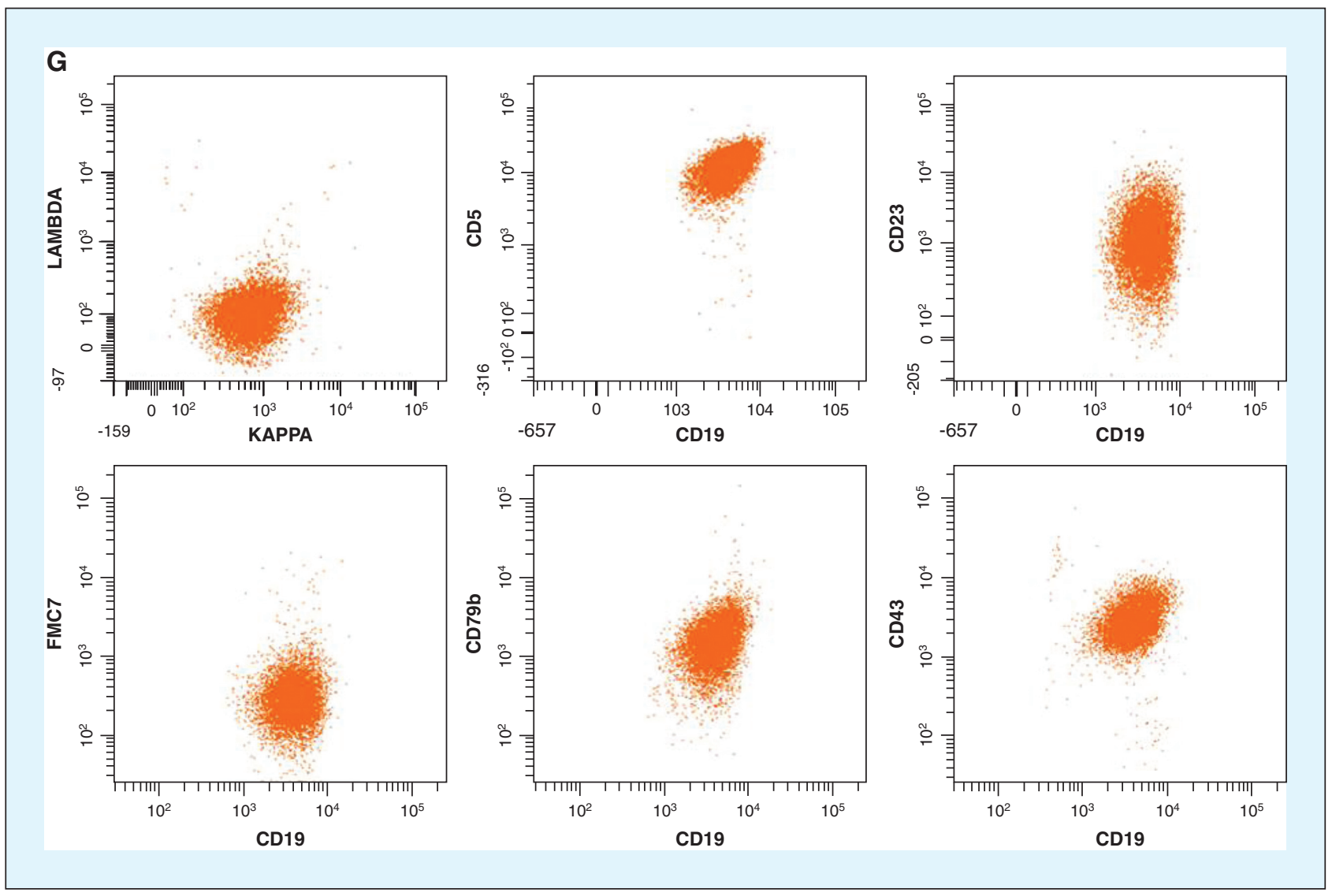

Figure 1. A , B, C : May-Grünwald Giemsa staining original magnification x100 ; D : myeloperoxidase staining by immunohistochemistry original magnification x100; E, F : electron microscopic aspect of intracytoplasmic inclusions (arrows), longitudinal section ; G : immunological profile with Matutes score $5 / 5$.

This observation is a reminder that the presence of Auer rod-shaped inclusions is in no way a myeloid malignancie.

Conflict of interest: none of the authors has any conflict of interest to disclose.

\section{References}

1. Oberley MJ, Yang DT. Lymphoproliferative disorder with Auer rod-like inclusions. Blood $2014 ; 16: 124(16): 2607$.

2. Hristov AC, Saladino A, Nava VE, Gocke CD. Auer rod-like inclusions in a low-grade B cell leukemia. Ann Diagn Pathol 2010;14: 292-5. 